\title{
Identification of Linear Power System Models Using Probing Signals
}

\author{
R. Cardenas-Javier, F. A. Zelaya, M. R. A. Paternina \\ National Autonomous University of Mexico - UNAM \\ \{rcardenasj,fzelaya223\}@ comunidad.unam.mx, mra.paternina@ fi-b.unam.mx
}

\author{
Felipe Wilches-Bernal \\ Sandia National Laboratories \\ fwilche@sandia.gov
}

\begin{abstract}
This paper compares the accuracy of two methods to identify a linear representation of a power system: the traditional Eigensystem Realization Algorithm (ERA) and the Loewner Interpolation Method (LIM). ERA is based on time domain data obtained using exponential chirp probing signals and LIM system identification method is based on frequency domain data obtained using sinusoidal probing signals. The ERA and LIM methods are evaluated with the noise produced by the nonlinear characteristics of the system, these characteristics are caused by increasing the amplitude of the applied probing signal. The test systems used are: the two-area Kundur system and a reduced order representation of the Northeastern portion of the North American Eastern Interconnection. The results show that the LIM method provides a more accurate identification than the ERA method.
\end{abstract}

\section{Introduction}

System identification methods estimate a linear model from measurement data of an actual system. Traditionally, some of the most important indicators of system identification methods are the accuracy of the estimated models as well as how immune to noise the methods are. For this comparison, the frequency response obtained with the small signal analysis (SSA) of the complete model of the system is used, the lack of an adequate complete model due to the various uncertainties of a system have motivated the comparison using different identification methods [1]. Classical methods use the impulse/pulse time domain response of the system [2], or assume that the transient response after a perturbation can be represented as unit impulse response. However, sometimes the measurements used to identify the model do not contain enough dynamic information because the applied pulse may not sufficiently excite the range of modes to be identified. Thus to improve the identification made with the Eigensystem Realization Algorithm (ERA) in [3] the system is modulated with an exponential chirp signal, this type of signal better excites the modes of interest, providing better selectivity to the identification.

The ERA method was initially developed in the aerospace community [4] but it has been successfully adapted and it is widely used in the power systems community $[5,6]$.

The Loewner Interpolation Method (LIM) was presented in [7] as a frequency-domain method to compute Frequency Dependent Network Equivalents (FDNEs) for electromagnetic transient (EMT) simulations. This method was first proposed by Antoulas et al. in [8] to generalize the identification problem, by fitting a descriptor system using sampling data from the transfer matrix of an actual system. The method has shown considerable advantages to modeling time domain macro-models from tabulated impedance, admittance or scattering parameters of Multiple-Input, Multiple Output (MIMO) systems [9, 10]. Furthermore, the LIM was recently used in [11] as an alternative for power system identification and model order reduction. However, that effort did not indicate the performance of the LIM method when noise is present in the measurements. This noise can be caused when the amplitude of the probing signal activates a limit in the nonlinear characteristics of the system. Therefore, in this article, the identification is carried out by gradually increasing the amplitude of the probing signal until the nonlinear characteristics of the system components are activated, then the system model is identified using ERA and LIM with similar criteria, and to evaluate which of these methods is more immune to noise.

On the other hand, in [11] large sets of modulation frequencies and extremely long simulation times are required. This paper shows how by grouping measurements using different modulated inputs, the accuracy of the identified linear system is considerably improved. This method allows for reducing the set of frequencies as well as the simulation times.

This paper is organized as follows. Section 2 summarizes ERA for system identification. Section 3 details the methodology of identification using LIM. Section 4 shows the results of using these system identification methods in two test power systems. Finally, Conclusions and Future Work are presented in Section 5. 


\section{The Eigensystem Realization Algorithm}

The ERA approach was introduced in [4] and is briefly explained in this section. Section 2.1 presents the formulation to identify the linear system model with a single input model. Section 2.2 presents the the extension of this formulation to identify the linear system model with a multiple input and multiple output model (MIMO). Section 2.3 describes the modulation of the inputs to obtain the impulse response and Section 2.4 presents the algorithm used for identification with ERA.

\subsection{Identification of Linear Models of SISO Systems with ERA}

The ERA system identification approach requires input and output data from the system. The output data usually is obtained from time-domain simulations or actual measurements taken from the system. Assuming a noiseless discrete-time input signal $(u(0), u(1), \cdots, u(N))$ that yields a zero-state response it is possible to generate the output signal as $[4,12]$ :

$$
\begin{aligned}
y(0) & =\mathbf{D} u(0) \\
y(1) & =\mathbf{C B} u(0)+\mathbf{D} u(1) \\
y(2) & =\mathbf{C A B} u(0)+\mathbf{C B} u(1)+\mathbf{D} u(2) \\
& \vdots \\
y(N-1) & =\mathbf{C A}^{N-1} \mathbf{B} u(0)+\cdots+\mathbf{C B} u(N-1)+\mathbf{D} u(N-1)
\end{aligned}
$$

where matrices $\mathbf{A}, \mathbf{B}, \mathbf{C}$, and $\mathbf{D}$ are known as the Markov parameters for the realization, and are assumed as unknown [12]. Such output sequence is generalized by

$$
y(k)=\mathbf{C A}^{k-1} \mathbf{B}
$$

Since the Markov parameters also define the linear time-invariant state-space model in discrete-time

$$
\begin{array}{r}
\mathbf{x}(k+1)=\mathbf{A x}(k)+\mathbf{B u}(k) \\
\mathbf{y}(k)=\mathbf{C x}(k)+\mathbf{D u}(k),
\end{array}
$$

then, the state vector in discrete-time is inferred by multiplying by $\mathbf{C}^{-1}$ the output sequence $\mathbf{y}(k)$ in (2) and assuming $\mathbf{D}=0$, which results in

$$
x(k)=\mathbf{A}^{k-1} \mathbf{B}
$$

Now, if the output sequence is defined by a Hankel representation, then we respectively have for $k$ and $k+1$

$$
\mathbf{H}(k)=\left[\begin{array}{cccc}
y(k) & y(k+1) & \cdots & y(k+N) \\
y(k+1) & y(k+2) & \cdots & y(k+N+1) \\
\vdots & \vdots & \ddots & \vdots \\
y(k+N) & y(k+N+1) & \cdots & y(k+2 N)
\end{array}\right]
$$

and

$$
\mathbf{H}(k+1)=\left[\begin{array}{cccc}
y(k+1) & y(k+2) & \cdots & y(k+N+1) \\
y(k+2) & y(k+3) & \cdots & y(k+N+2) \\
\vdots & \vdots & \ddots & \vdots \\
y(k+N+1) & y(k+N+2) & \cdots & y(k+2 N+1)
\end{array}\right]
$$

which in terms of the generalized output sequence is given by

$$
\mathbf{H}(k)=\left[\begin{array}{cccc}
\mathbf{C A}^{k-1} \mathbf{B} & \mathbf{C A}^{k} \mathbf{B} & \cdots & \mathbf{C A}^{k-1+n} \mathbf{B} \\
\mathbf{C A}{ }^{k} \mathbf{B} & \mathbf{C A}^{k+1} \mathbf{B} & \cdots & \mathbf{C A}^{k+n} \mathbf{B} \\
\vdots & \vdots & \ddots & \vdots \\
\mathbf{C A}^{k-1+n} \mathbf{B} & \mathbf{C A}^{k+n} \mathbf{B} & \cdots & \mathbf{C A}^{k-1+2 n} \mathbf{B}
\end{array}\right]
$$

The generalized Hankel representation (7) in matrix form then becomes

$$
\mathbf{H}(k)=\underbrace{\left[\begin{array}{c}
\mathbf{C} \\
\mathbf{C A} \\
\vdots \\
\mathbf{C A}^{n}
\end{array}\right]}_{\boldsymbol{\xi}} \mathbf{A}^{k-1}\left[\begin{array}{lll}
\mathbf{B} & \mathbf{A B} & \cdots
\end{array} \mathbf{A}^{n} \mathbf{B}\right]
$$

where $\boldsymbol{\xi}$ in (8) stands for the observability and the term $\mathbf{A}^{k-1} \mathbf{B}$ represents the controllability. If only $k=1$ and $k=2$ are taken into account in (8), then we have

$$
\begin{aligned}
& \mathbf{H}(1)=\boldsymbol{\xi} \mathbf{B} \\
& \mathbf{H}(2)=\boldsymbol{\xi} \mathbf{A B}
\end{aligned}
$$

Finally, it is possible to estimate the Markov parameters from (9) by means of the Singular Value Decomposition (SVD) applied to $\mathbf{H}(1) \in \mathbb{R}^{N \times N}$ and its truncation up to the $r^{\text {th }}$ singular value (with $r<N$ ), ensuring a full range for the system and is given by.

$$
\frac{\sum_{l=1}^{r} \sigma_{l}}{\sum_{l=1}^{N} \sigma_{l}} \leq E_{\text {era }}
$$

where $l=1,2,3, \ldots N . \forall r \leq N$ and $E_{\text {era }}$ is the energy criterion which is user-defined, $r$ is the order of the truncated system, and $\sigma$ refers to the $N$ singular values of the system. This can be written as $\mathbf{H}(1)=$ $\mathbf{P S}^{1 / 2} \mathbf{S}^{1 / 2} \mathbf{Q}^{T}$, which derives that $\boldsymbol{\xi}=\mathbf{P S}^{1 / 2}$ and $\mathbf{B}=\mathbf{S}^{1 / 2} \mathbf{Q}^{T}$ in (9), leading to estimate the discrete system matrices as:

$$
\begin{aligned}
& \mathbf{A}=\mathbf{S}^{-1 / 2} \mathbf{P}^{T} \mathbf{H}(2) \mathbf{Q} \mathbf{S}^{-1 / 2} \\
& \mathbf{B}=\mathbf{S}^{1 / 2} \mathbf{Q}^{T} \\
& \mathbf{C}=\mathbf{P} \mathbf{S}^{1 / 2} \\
& \mathbf{D}=y(0)
\end{aligned}
$$




\subsection{Identification of Linear Models of MIMO Systems with ERA}

For multiple outputs channels, the matrix $\mathbf{Y}_{i} \in$ $\mathbb{R}^{p \times m}$ is shaped by $m$ column arrays corresponding to single channels as follows

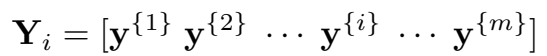

where the $i^{\text {th }}$ column is represented by $\mathbf{y}^{\{i\}}=$ $\left[\begin{array}{llll}y_{1} & y_{2} & \cdots & y_{p}\end{array}\right]^{T}$. Similar to (1), the input/output measurement pairs to an known input allow to express the output sequence for multiple channels as

$$
\begin{aligned}
\mathbf{Y}_{0}= & \tilde{\mathbf{D}} \\
\mathbf{Y}_{1} & =\tilde{\mathbf{C}} \tilde{\mathbf{B}} \\
\mathbf{Y}_{2}= & \tilde{\mathbf{C}} \tilde{\mathbf{A}} \tilde{\mathbf{B}} \\
& \vdots \\
\mathbf{Y}_{N-1} & =\tilde{\mathbf{C}} \tilde{\mathbf{A}}^{N-1} \tilde{\mathbf{B}}
\end{aligned}
$$

It is worth noting that (13) follows the same sequence than (2) for the Markov parameters of multiple channels termed as $\tilde{\mathbf{A}}, \tilde{\mathbf{B}}, \tilde{\mathbf{C}}$, and $\tilde{\mathbf{D}}$. Thereby, the Hankel matrix can be also stated for multiple output channels as $\tilde{\mathbf{H}}(k)=\tilde{\boldsymbol{\xi}} \tilde{\mathbf{A}}^{k-1} \tilde{\mathbf{B}}$. Afterwards, the block Hankel matrix in (5) becomes as

$$
\tilde{\mathbf{H}}(k)=\left[\begin{array}{cccc}
\mathbf{Y}_{k} & \mathbf{Y}_{k+1} & \cdots & \mathbf{Y}_{k+N} \\
\mathbf{Y}_{k+1} & \mathbf{Y}_{k+2} & \cdots & \mathbf{Y}_{k+N+1} \\
\vdots & \vdots & \ddots & \vdots \\
\mathbf{Y}_{k+N} & \mathbf{Y}_{k+N+1} & \cdots & \mathbf{Y}_{k+2 N}
\end{array}\right]
$$

By assuming $k=1$ and $k=2$ into (14), the Hankel matrices $\tilde{\mathbf{H}}(1)$ and $\tilde{\mathbf{H}}(2)$ can be derived as in (9).

Now, $\tilde{\mathbf{B}}$ can be obtained from $\tilde{\mathbf{H}}(1) \in \mathbb{R}^{p(N) \times m(N)}$, and $\tilde{\mathbf{A}}$ is derived from $\tilde{\mathbf{H}}(2) \in \mathbb{R}^{p(N) \times m(N)}$, its truncation up to the $r^{\text {th }}$ singular value (with $r<N$ ), ensuring a full range for the system and is given by (10). Therefore, the Markov parameters for multiple output channels have the following form:

$$
\begin{aligned}
\tilde{\mathbf{A}} & =\tilde{\mathbf{S}}^{-1 / 2} \tilde{\mathbf{P}}^{T} \tilde{\mathbf{H}}(2) \tilde{\mathbf{Q}} \tilde{\mathbf{S}}^{-1 / 2} \\
\tilde{\mathbf{B}} & =\tilde{\mathbf{S}}^{1 / 2} \tilde{\mathbf{Q}}^{T} \\
\tilde{\mathbf{C}} & =\tilde{\mathbf{P}} \tilde{\mathbf{S}}^{1 / 2} \\
\tilde{\mathbf{D}} & =\mathbf{Y}_{0}
\end{aligned}
$$

After the Markov parameters in (3) are estimated in discrete time, the continuous-time state-space model can be readily obtained by using simple matrix transformations ${ }^{1}$. In this work this transformation is performed using the $\mathrm{d} 2 \mathrm{c}$ Matlab function.

\subsection{Modulating the Inputs of the System with Probing Signals}

This work uses a frequency sweep function to excite the inputs of the system. This signal would be the input signal used by the ERA approach. The probing signal selected to modulate the inputs and excite the system dynamics is an exponential chirp function defined by [13]

$$
u_{i}(t)=\alpha_{i} \sin \left(\frac{2 \pi f_{s}\left(r_{f}^{t}-1\right)}{\ln \left(r_{f}\right)}\right)
$$

with

$$
r_{f}=\left(\frac{f_{e}}{f_{s}}\right)^{1 / T}
$$

where $\alpha_{i}$ is the amplitude, $T$ is the duration, $r_{f}$ is the rate of frequency, $f_{s}$ and $f_{e}$ are, respectively, the starting and ending frequencies.

The output signals required by the ERA approach were generated using time-domain simulations when the inputs of the system were modulated by chirp signals. Afterwards, Fourier spectral analysis is applied using fft to inputs $\mathscr{F}\left(u_{i}(t)\right)$ and outputs, $\mathscr{F}\left(y_{q, i}^{\prime}(t)\right)$, resulting in the frequency response $U_{i}(\omega)$ and $Y_{q, i}^{\prime}(\omega)$, respectively.

Finally, a time output sequence per signal correlated with the $i^{\text {th }}$ input is obtained taking the inverse Fourier transform of the impulse response as [13]

$$
\mathbf{y}_{q, i}(t)=\mathscr{F}^{-1}\left(\frac{Y_{q, i}^{\prime}(\omega)}{U_{i}(\omega)}\right)
$$

Filters can be used to reduce the signal's noise.

\subsection{Algorithm to Identification with ERA}

The responses of the system outputs $y_{q, i}^{\prime}$ resulting from the modulation in the input $u_{i}$ with the exponential chirp function, are needed to perform the ERA system identification using Algorithm 1. The steps in this approach are: (i) Modulating the Inputs to obtain impulse response; (ii) Hankel matrix assembling; and (iii) Right-order selection and model extraction [13].

\footnotetext{
${ }^{1}$ For example the relationship between discrete and continuous matrices is $A_{\text {discrete }}=e^{A_{\text {continuous }} T_{s}}$, where $T_{s}$ is the sampling time.
} 


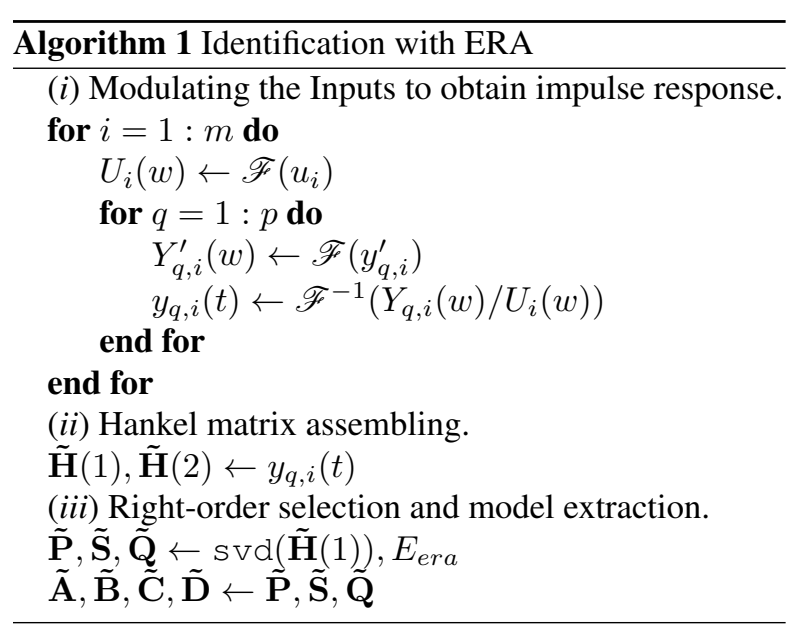

\section{The Loewner Interpolation Method}

The Loewner Frequency Interpolation method uses frequency-domain data to obtain the realization of the system. This method for system identification uses modulation of selected inputs and the measurement of selected outputs. This data is used to fill the Loewner matrices, which are used to interpolate the frequency data and obtain the set of matrices A, B, C, D.

Let $\mathbf{H}\left(s_{k}\right)$ be the transfer function at the $k^{\text {th }}$ frequency that represents a MIMO system defined as

$$
\mathbf{H}\left(s_{k}\right)=\frac{\mathbf{Y}\left(s_{k}\right)}{\mathbf{U}\left(s_{k}\right)}
$$

where $\mathbf{Y}\left(s_{k}\right)$ and $\mathbf{U}\left(s_{k}\right)$ refer to the outputs and inputs of the system, respectively. The Loewner's process starts by getting the frequency data $\mathbf{H}\left(s_{k}\right)$. This is accomplished by measuring the frequency-response when several inputs of the system are modulated at the $k^{\text {th }}$ frequency $s_{k}$. Considering a selected frequency range in the interval $\left[s_{\min }, s_{\max }\right]$, it is possible to obtain a linear representation in this range. This representation that ensures a minimal realization representing the dynamics of the system is given by $[9,11,14]$.

$$
\begin{aligned}
\mathbf{E}_{r} \dot{\mathbf{x}}(t) & =\mathbf{A}_{r} \mathbf{x}(t)+\mathbf{B}_{r} \mathbf{u}(t) \\
\mathbf{y}(t) & =\mathbf{C}_{r} \mathbf{x}(t)+\mathbf{D}_{r} \mathbf{u}(t)
\end{aligned}
$$

The interpolation process for system identification consist in three stages summarized as: (i) data assembling, (ii) data grouping and interpolations (iv) right-order selection and model extraction.

\subsection{Probing Signals for Input Modulation and Extraction of Frequency Response}

To obtain the frequency response $\mathbf{H}\left(s_{k}\right)$, the system should be excited with some low-level time-domain linear inputs. Sinusoidal test signals are set up as reference of the form $\mathbf{u}_{i}=\alpha \sin \left(2 \pi f_{k} t+\varphi_{0}\right)$, where $i$ is the index of the selected inputs to modulate, $\alpha$ is the amplitude of the modulation signal and in practice tend to be small, $f_{k}$ is the frequency of interest, and $\varphi_{0}$ is the phase of the input signals. The system is modulated one input at a time for every frequency point proposed in the selected frequency range.

A transfer function $\mathbf{H}\left(s_{k}\right)$ is constructed with the maximum frequency component of each output $\mathscr{F}\left(y_{q, i}\right)$ given by $\hat{y}_{q, i}+\beta_{q, i} \sin \left(2 \pi f_{k} t+\theta_{q, i}\right)+\eta_{q, i}$ as well as by the maximum frequency component of each input $\mathscr{F}\left(u_{i}\right)$ given by $\alpha_{i} \sin \left(2 \pi f_{k} t+\phi_{i}\right)$. Then, the output measurements and the input probing signals are correlated by

$$
\mathbf{H}\left(s_{k}\right)=\frac{\beta_{q, i}}{\alpha_{i}} e^{j\left(\theta_{q, i}-\phi_{i}\right)}
$$

where the tensor $\mathbf{H}\left(s_{k}\right) \in \mathbb{R}^{p \times m \times \Gamma}$ and $s_{k}=j 2 \pi f_{k}$.

\subsection{Data Grouping and Interpolation}

Because the frequency-domain data is available in the form $\left[s_{k}, \mathbf{H}\left(s_{k}\right)\right]$, the method requires dividing it into two different sub-groups and enabling the interpolation among them. Thus, the actual data are appended with their complex conjugates at the negative frequencies, resulting $2 \Gamma$ sample points:

$$
\left[s_{k}, \mathbf{H}\left(s_{k}\right)\right] \cup\left[-s_{k}, \mathbf{H}^{H}\left(s_{k}\right)\right] \rightarrow\left[s_{l}, \mathbf{H}\left(s_{l}\right)\right]
$$

where (.) ${ }^{H}$ denotes the complex conjugate and $1 \leqslant l \leqslant$ $2 \Gamma$. Then, the data is divided as follows

$$
\left[s_{l}, \mathbf{H}\left(s_{l}\right)\right] \rightarrow\left[\lambda_{i}, \mathbf{H}\left(\lambda_{i}\right)\right],\left[\mu_{j}, \mathbf{H}\left(\mu_{j}\right)\right]
$$

where $\left[\lambda_{i}, \mathbf{H}\left(\lambda_{i}\right)\right]$ and $\left[\mu_{j}, \mathbf{H}\left(\mu_{j}\right)\right]$ are termed the left and right data, $, \forall i, j=1,2, \ldots, \Gamma$. Despite there are different forms to group the data, the recent literature focuses on two different approaches [8, 10, 15]: Vector Format Tangential Interpolation (VFTI) and Matrix Format Tangential Interpolation (MFTI). Choosing the MFTI approach, the frequencies are divided into odd and even, shaping right data as,

$$
\begin{aligned}
\lambda_{2 i-1} & =s_{2 i-1} \\
\lambda_{2 i} & =\bar{s}_{2 i-1}
\end{aligned}
$$


and, the left data are as

$$
\begin{aligned}
\mu_{2 i-1} & =s_{2 i} \\
\mu_{2 i} & =\bar{s}_{2 i}
\end{aligned}
$$

where $i=1,2, \ldots, \Gamma / 2$. Note that the values associated with $\mathbf{H}\left(s_{k}\right)$ are also divided following the same relationship as the frequency points $s_{k}$.

The frequency interpolation is obtained by employing the Loewner matrices to make use of the result of the data grouping and directionality stages. In this way, the matrix Loewner $\mathbb{L}$, shifted Loewner matrix $\sigma \mathbb{L}$, together with matrices $\mathbb{F}$ and $\mathbb{W}$, can be defined following the principles of the Loewner method in [9] and taking into account the input and output parametric matrices of the system. Thus, these Loewner matrices are assembled as

$$
\begin{aligned}
& \mathbb{L}_{j, i}=\left[\begin{array}{ccc}
\frac{\mathbf{H}\left(\mu_{1}\right)-\mathbf{H}\left(\lambda_{1}\right)}{\mu_{1}-\lambda_{1}} & \cdots & \frac{\mathbf{H}\left(\mu_{1}\right)-\mathbf{H}\left(\lambda_{i}\right)}{\mu_{1}-\lambda_{i}} \\
\vdots & \ddots & \vdots \\
\frac{\mathbf{H}\left(\mu_{j}\right)-\mathbf{H}\left(\lambda_{1}\right)}{\mu_{j}-\lambda_{1}} & \cdots & \frac{\mathbf{H}\left(\mu_{j}\right)-\mathbf{H}\left(\lambda_{i}\right)}{\mu_{j}-\lambda_{i}}
\end{array}\right] \\
& \sigma \mathbb{L}_{j, i}=\left[\begin{array}{ccc}
\frac{\mu_{1} \mathbf{H}\left(\mu_{1}\right)-\lambda_{1} \mathbf{H}\left(\lambda_{1}\right)}{\mu_{1}-\lambda_{1}} & \cdots & \frac{\mu_{1} \mathbf{H}\left(\mu_{1}\right)-\lambda_{i} \mathbf{H}\left(\lambda_{i}\right)}{\mu_{1}-\lambda_{i}} \\
\vdots & \ddots & \vdots \\
\frac{\mu_{j} \mathbf{H}\left(\mu_{j}\right)-\lambda_{1} \mathbf{H}\left(\lambda_{1}\right)}{\mu_{j}-\lambda_{1}} & \cdots & \frac{\mu_{j} \mathbf{H}\left(\mu_{j}\right)-\lambda_{i} \mathbf{H}\left(\lambda_{i}\right)}{\mu_{j}-\lambda_{i}}
\end{array}\right] \\
& \mathbb{F}=\left[\mathbf{H}\left(\mu_{1}\right)^{T}, \cdots, \mathbf{H}\left(\mu_{j}\right)^{T}, \cdots, \mathbf{H}\left(\mu_{N}\right)^{T}\right]^{T} \\
& \mathbb{W}=\left[\mathbf{H}\left(\lambda_{1}\right), \cdots, \mathbf{H}\left(\lambda_{i}\right), \cdots, \mathbf{H}\left(\lambda_{N}\right)\right]
\end{aligned}
$$

where $[\mathbb{L}, \sigma \mathbb{L}] \in \mathbb{C}^{p(\Gamma) \times m(\Gamma)}, \mathbb{F} \in \mathbb{C}^{p(\Gamma) \times m}$, and $\mathbb{W} \in \mathbb{C}^{p \times m(\Gamma)}$. Given that matrices (26) - (29) are complex and they are fitting a real model, they have to be transformed to the real form by means of the similarity transformation [9], as follows:

$$
\begin{array}{rr}
\mathbb{L}_{\mathrm{Re}}=\mathbb{G}^{*} \mathbb{L} \mathbb{G}, & \sigma \mathbb{L}_{\mathrm{Re}}=\mathbb{G}^{*} \sigma \mathbb{L} \mathbb{G} \\
\mathbb{F}_{\mathrm{Re}}=\mathbb{G}^{*} \mathbb{F}, & \mathbb{W}_{\mathrm{Re}}=\mathbb{W} \mathbb{G}
\end{array}
$$

where $\mathbb{G} \in \mathbb{C}^{\Gamma \times \Gamma}$ is a block diagonal matrix formed by several sub-matrices $\Upsilon$, given by

$$
\mathbf{\Upsilon}=\frac{1}{\sqrt{2}}\left[\begin{array}{cc}
\mathbf{I} & -j \mathbf{I} \\
\mathbf{I} & j \mathbf{I}
\end{array}\right]
$$

where $\mathbf{I} \in \mathbb{R}^{t_{i} \times t_{i}}$ is the identity matrix.

\subsection{Right-order Selection and Model Extraction}

Since the realization of the system is not unique, the method needs to guarantee the minimal realization. This refers to the smallest possible order $m$ of the system [10], that ensures a correct identification of the system along the frequency range of concern. The $r^{\text {th }}$ order is determined by performing a singular value decomposition (SVD) on the Loewner matrix pencil, as follows

$$
x \mathbb{L}_{\mathrm{Re}}-\sigma \mathbb{L}_{\mathrm{Re}}=\Lambda \Sigma \Psi^{*}
$$

where $x \in\left\{\lambda_{i}\right\} \cup\left\{\mu_{i}\right\}$, and $\lambda_{i}, \mu_{i} \notin \operatorname{eig}\left(\sigma \mathbb{L}_{\mathrm{Re}}, \mathbb{L}_{\mathrm{Re}}\right), \boldsymbol{\Sigma}$ is the diagonal matrix that contains the singular values of the system, $\Lambda$ and $\boldsymbol{\Psi}$ are orthonormal matrices, and ()$^{*}$ refers to the complex conjugated transpose $[8,9]$.

The order $r$ for the identified system is given by,

$$
\frac{\sum_{t=1}^{r} \sigma_{l}}{\sum_{t=1}^{p \times \Gamma} \sigma_{l}} \leq E_{l i m}
$$

where $t=1,2,3, \ldots(p \times \Gamma) . \forall r \leq(p \times \Gamma)$ and $E_{\text {lim }}$ is the energy criterion which is user-defined, $r$ is the order of the system, and $\sigma$ refers to the $p \times \Gamma$ singular values of the system.

Subsequently, the model extraction in time-domain is carried out by reducing the columns of the matrices $[\Lambda, \Psi]$, and considering the order obtained as $r$, allowing to get matrices of the $r^{\text {th }}$ order $\left[\Lambda_{r}, \Psi_{r}\right]$, whose columns are used as projectors to extract the regular part of the system, as follows

$$
\begin{aligned}
& \mathbf{E}_{r}^{\prime}=-\Lambda_{r}^{*} \mathbb{L}_{\operatorname{Re}} \Psi_{r} \\
& \mathbf{A}_{r}^{\prime}=-\Lambda_{r}^{*} \sigma \mathbb{L}_{\operatorname{Re}} \Psi_{r} \\
& \mathbf{B}_{r}^{\prime}=\Lambda_{r}^{*} \mathbb{F}_{\operatorname{Re}} \\
& \mathbf{C}_{r}^{\prime}=\mathbb{W}_{\operatorname{Re}} \Psi_{r} \\
& \mathbf{D}_{r}^{\prime}=0
\end{aligned}
$$

Finally, the parameters of linear model is given by $\mathbf{A}_{\mathrm{r}}=\mathbf{E}^{\prime-1} \mathbf{A}^{\prime}, \mathbf{B}_{\mathrm{r}}=\mathbf{E}^{\prime-1} \mathbf{B}^{\prime}, \mathbf{C}_{\mathrm{r}}=\mathbf{C}_{r}^{\prime}$ and $\mathbf{D}_{r}=0$.

\subsection{Algorithm to Identification with LIM}

Obtained the responses of outputs system $y_{q, i, k}(t)$ resulting from the inputs modulation $u_{i, k}(t)$ with sinusoidal function. Algorithm 2 show the main steps of LIM according based on [9], they are: (i) Extraction of frequency response and assembling; (ii) Grouping and interpolation; (iii) Right-order selection and system realization. 


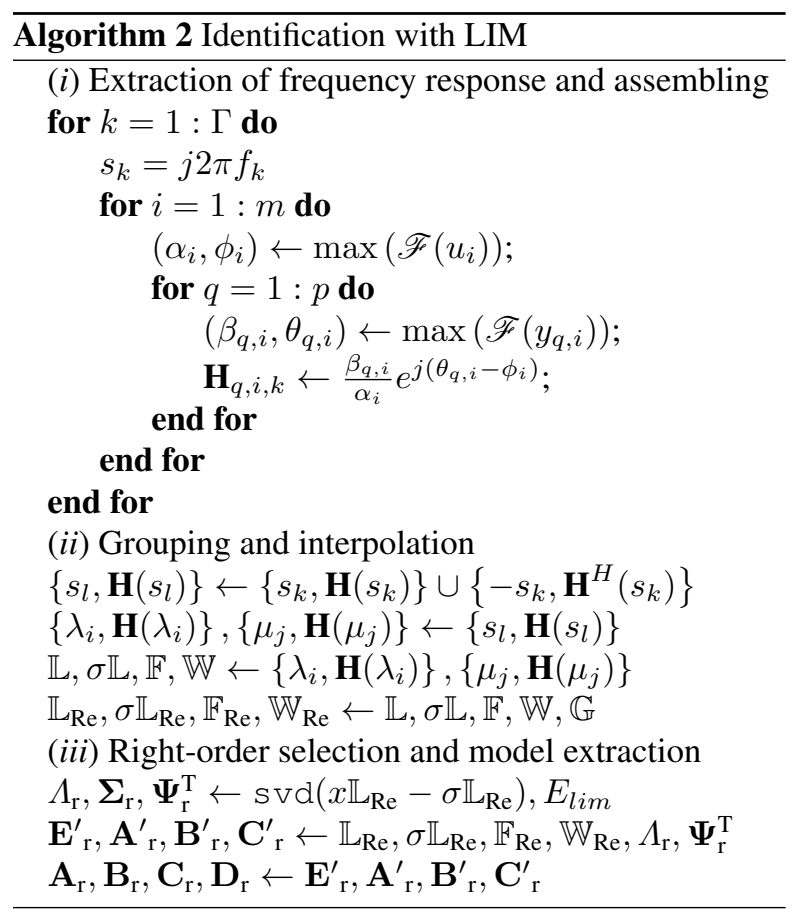

\section{Performance Comparison}

This section presents a comparison of the performance of the methods to identify the parameters of linear model in power systems, ERA presented in the Section 2 and LIM presented in 3. The results of two-area KRK system [16] are presented in Section 4.1 while the results for a system representative of the Northeastern part of the North American Eastern Interconnection [17] are presented in Section 4.2.

The data of the transient simulations applying the probing signals are obtained using the routine S_simu of the software Power System Toolbox (PST) [18]. As indicated in [13], to use the exponential chirp modulation, the probing signal has the following parameters $f_{s}=0.1$ to $f_{e}=3.0 \mathrm{~Hz}$ and $T=56 \mathrm{~s}$. Likewise, to use LIM method, this work proposes $68 \mathrm{~s}$ of sinusoidal signals with ten frequencies between 0.1 to $3.0 \mathrm{~Hz}$ spaced with (35):

$$
f_{k}=\left\{\begin{array}{c}
0.10+0.25(k-1), \quad 1 \leq k \leq 7 \\
2.00+0.50(k-8), \quad 8 \leq k \leq 10
\end{array}\right.
$$

The accuracy of the identified systems is determined by comparing them with the ideal linearized system around an operating point obtained with PST (using the routine svm mgen), this function performs a small signal analysis (SSA) of complete equations of the power system, therefore the parameters $\mathbf{A}, \mathbf{B}, \mathbf{C}$ and $\mathbf{D}$ are considered as a real linear model of the system.
It is important to note that a change in the operating point of the system changes the parameters of the state space model, therefore, the probing signal applied to the inputs of the electrical system should have a short duration like the one proposed in this work.

\subsection{KRK: Two-area, Four-machine System}

The KRK system has two local modes and one inter-area mode; the system used in this work has 48 states, 12 for each generator (6 states for the synchronous machine, 3 states for the DC exciter and 3 states for the turbine-governor). All generators can be modulated, the system inputs are the voltage reference in the DC exciter indicated by (Vr) and the scheduled mechanical power indicated by $(\mathrm{Pm})$. The outputs of the system are the rotor speeds indicated by $(\omega)$. Fig. 1 shows the inputs of KRK, the exponential chirp and the set of sinusoidal signals.

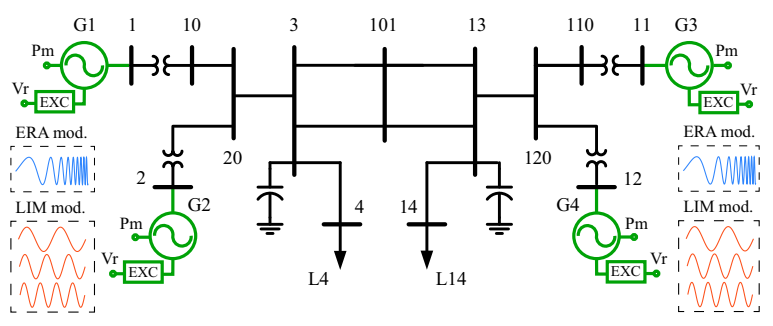

Figure 1. KRK system and modulation signal used.

Using the KRK system, it is evaluated how the precision of the identified system is maintained against the noise produced by the nonlinear characteristics of the system caused by gradually increasing the magnitude of the probing signal $\alpha_{i}$. To do this, first an identification is carried out with ERA and LIM is obtained with $\alpha_{i}=5 \%$ using the $\mathrm{Vr}$ input and the $\omega_{i}$ output, then it is increased 4 times $9 \%$ until reaching $\alpha_{i}=41 \%$ of the reference value. In Fig. 2 shows the comparison of the different probing signals and the magnitude of the Bode plot of the identifications made to these measurements, in Fig. 2(a) and Fig. 2(b) shows the output $\omega_{1}$ modulating the input $V r_{1}$ with exponential chirp and sinusoidal function, respectively. Furthermore, in Fig. 2(c) and Fig. 2(d) shows the magnitude of the Bode of the transfer function $\omega_{1} / V r_{1}$ identified with ERA and LIM, respectively.

For both identification methods the same energy criteria is used: $E_{\text {era }}=E_{\text {lim }}=99 \%$. In order to clearly analyze the accuracy of each method, Table 1 presents the increase in the root-mean-square error (RMSE) of the magnitude of the identification in the frequency band 0.1 to $50 \mathrm{rad} / \mathrm{s}$ when the amplitude of the probing signal is gradually increased. 
Table 1. RMSE of KRK system identification by increasing the amplitude of the probing signal.

\begin{tabular}{c|c|c|c|c|c}
\hline Method & $\alpha=0.05$ & $\alpha=0.14$ & $\alpha=0.23$ & $\alpha=0.32$ & $\alpha=0.41$ \\
\hline ERA & $6.63 \times 10^{-3}$ & $6.81 \times 10^{-3}$ & $14.99 \times 10^{-3}$ & $15.51 \times 10^{-3}$ & $18.37 \times 10^{-3}$ \\
LIM & $1.16 \times 10^{-3}$ & $1.71 \times 10^{-3}$ & $3.19 \times 10^{-3}$ & $3.76 \times 10^{-3}$ & $4.03 \times 10^{-3}$ \\
\hline
\end{tabular}
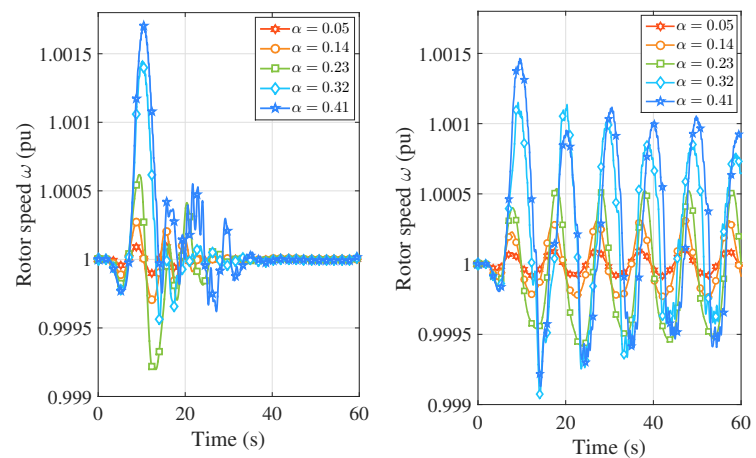

(a) $\omega_{1}$ increasing $\alpha_{1}$ of the (b) $\omega_{1}$ increasing $\alpha_{1}$ of the exponential chirp.
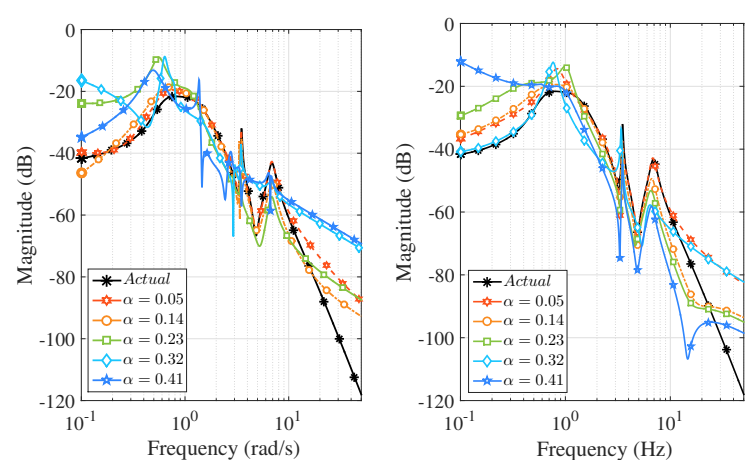

(c) Bode of $\omega_{1}(s) / V r_{1}(s)$ using (d) Bode of $\omega_{1}(s) / V r_{1}(s)$ ERA method.

using LIM method.

Figure 2. KRK accuracy of ERA and LIM methods by increasing the amplitude of the probing signal.

As can be seen, the LIM method is more precise despite the noise produced by the nonlinear characteristics of the system, it can also be observed that at the amplitudes 0.05 and 0.14 both methods are quite precise, but with the amplitude of $0.23,0.32$ and 0.41 the ERA method worsens faster than LIM.

On the other hand, the frequency range and effective simulation time presented captures local and inter-area modes well but is slightly less accurate than that performed with a wide set of frequencies and extremely long simulation times. To improve this identification, this work presents the improvement in the identification performance using the multiple grouping of measurements. In Table 2 shows the size of the matrices for identification using ERA and LIM in the KRK system in simple identifications with $\mathrm{Vr}$ or Pm and using grouping of $\mathrm{Vr}$ and Pm measurements (multiple).
Table 2. ERA and LIM matrices in KRK system.

\begin{tabular}{c|c|c|c|c|c|c}
\hline Mod. & $\mathbb{L}_{r}, \sigma \mathbb{L}_{r}$ & $\mathbb{F}_{r}$ & $\mathbb{W}_{r}$ & Order & $\tilde{\mathbf{H}}(1), \tilde{\mathbf{H}}(2)$ & Order \\
\hline $\mathrm{Vr}$ & $40 \times 40$ & $40 \times 4$ & $4 \times 40$ & 9 & $480 \times 480$ & 12 \\
\hline $\mathrm{Pm}$ & $480 \times 480$ & $40 \times 4$ & $4 \times 40$ & 13 & $13 \times 13$ & 12 \\
\hline $\mathrm{Vr}+\mathrm{Pm}$ & $40 \times 80$ & $40 \times 8$ & $4 \times 80$ & 14 & $4 \times 22$ & 22 \\
\hline
\end{tabular}

Fig. 3 shows the eigenvalues of the identified system for the KRK model. Figs. 3(a) and 3(b) Pm show the results of using individual measurements, $\mathrm{Vr}$ an $\mathrm{Pm}$, respectively. Fig. 3(c) shows the eigenvalues when the the two sets of measurements, $\mathrm{Vr}$ and Pm are used in the system identification approach. For this latter case the inter-area and local modes identified are closer to the actual values that for any of the cases in Figs. 3(a) and 3(b).

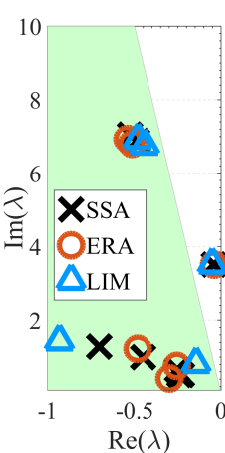

(a) Simple Vr

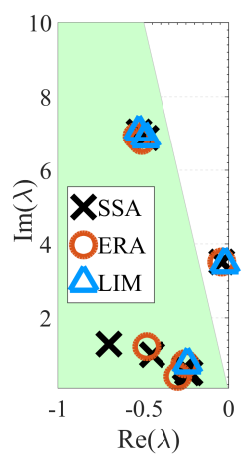

(b) Simple Pm

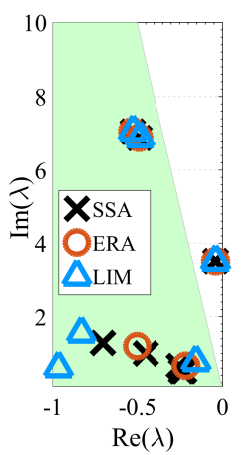

(c) Multiple Vr, Pm
Figure 3. KRK eigenvalues grouping measurements.

In Fig. 4 shows the Bode diagram of identified linear systems, using single and multiple data, in Fig. 4(a) shows $\omega_{1} / V r_{1}$ and in Fig. 4(b) shows $\omega_{1} / P m_{1}$.

To observe the increase in precision in Table 3 presents the RMSE of the identifications made with multiple measurements, $\omega_{1} / V r_{1}$ with LIM it presents an error of $1.84 \times 10^{-3}, 49 \%$ less than ERA method. Likewise, for $\omega_{1} / P m_{1}$ presents an error $1.17 \times 10^{-3}$ is $41 \%$ less than ERA method.

\subsection{NPCC: 48 Generators and 140 Bus System}

The NPCC power system [17, 19] represents one of the eight regional reliability organizations that are part of NERC. The model used of NPCC system has 

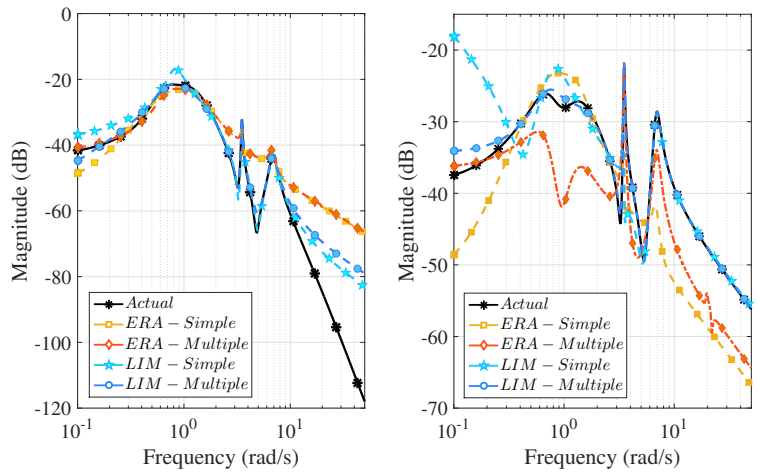

(a) $\omega_{1} / V r_{1}$ Simple and Multiple (b) $\omega_{1} / P m_{1}$ Simple and Multiple

Figure 4. KRK precision of ERA and LIM methods by grouping measurements.

Table 3. Identification accuracy in KRK system.

\begin{tabular}{c|c|c|c}
\hline Transfer function & Method & Simple & Multiple \\
\hline \multirow{2}{*}{$\omega_{1}(s) / V r_{1}(s)$} & ERA & $4.22 \times 10^{-3}$ & $4.07 \times 10^{-3}$ \\
\cline { 2 - 4 } & LIM & $6.30 \times 10^{-3}$ & $1.84 \times 10^{-3}$ \\
\hline \multirow{2}{*}{$\omega_{1}(s) / P m_{1}(s)$} & ERA & $11.18 \times 10^{-3}$ & $10.08 \times 10^{-3}$ \\
\cline { 2 - 4 } & LIM & $6.62 \times 10^{-3}$ & $1.17 \times 10^{-3}$ \\
\hline
\end{tabular}

48 generators and for the identification it is considered that only 24 generators are enabled to be modulated, and those can be observed by the green dots in Fig. 5 .

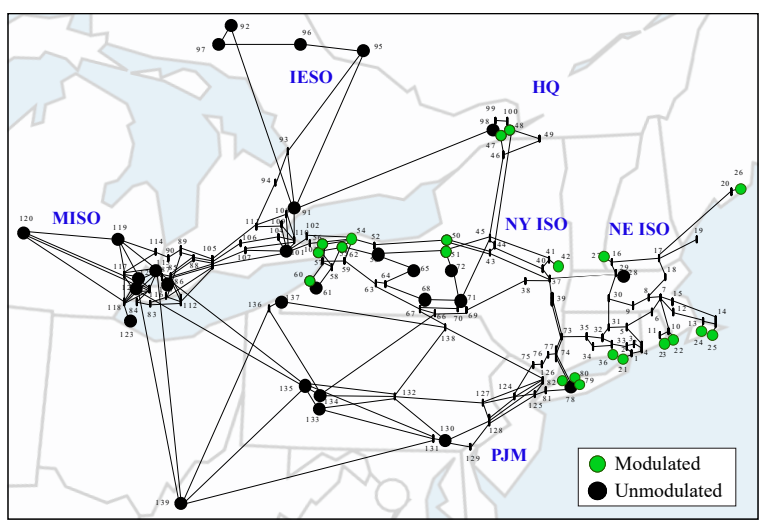

Figure 5. NPCC power system identified.

The NPCC system used in this work has 294 states. 27 of the total number of generators are represented by four states (transient model) and the remaining 21 generators are represented by two states (electromechanical model). For the 24 enabled generators, there are additional three states for the DC exciter and three states for the turbine-governor.

Using the NPCC system, it is evaluated how the accuracy of the identified linear system is maintained against the noise produced by the nonlinear characteristics caused by gradually increasing of the magnitude of the probing signal $\alpha_{i}$. As in the previous case, first an identification is carried out with ERA and LIM is obtained with $\alpha_{1}=5 \%$ using the $\mathrm{Vr}$ as the input and the $\omega_{1}$ as the output, then it is increased 4 times $9 \%$ until reaching $\alpha_{i}=41 \%$ of the reference value.

Fig. 6 shows the comparison of magnitude of the Bode plot of the identifications models with different values of $\alpha_{1}$, in Fig. 6(a) and Fig. 6(b) shows the magnitude of the Bode of the transfer function $\omega_{1} / V r_{1}$ identified with ERA and LIM, respectively.
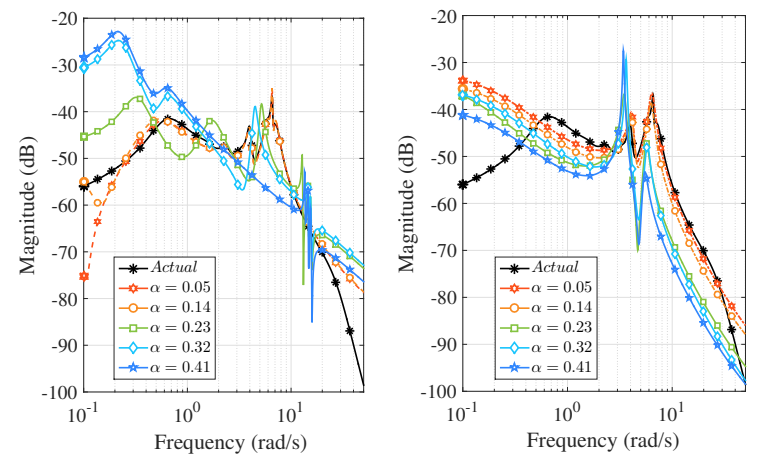

(a) Bode of $\omega_{1}(s) / V r_{1}(s)$ using (b) Bode of $\omega_{1}(s) / V r_{1}(s)$ ERA method. using LIM method.

Figure 6. NPCC accuracy of ERA and LIM methods by increasing the amplitude of the probing signal.

For both identification methods the same energy criteria are used, $E_{\text {era }}=E_{l i m}=90 \%$, to clearly analyze the precision of each method, Table 4 presents the increase in error (Root-mean square error - RMSE) of the magnitude of the identification in the frequency band 0.1 to $50 \mathrm{rad} / \mathrm{s}$ when the amplitude of the probing signal is gradually increased.

As can be seen, the LIM method is more accurate despite the noise produced by the nonlinear characteristics of the system with all amplitudes of $\alpha_{1}$, but the ERA method only maintains precision with amplitudes of 0.05 and 0.14 , losing precision with amplitudes $0.23,0.32$ and 0.41 .

As in the previous case, the improvement of the precision of the linear identification of the model is presented by grouping multiple measurements. In Table 5 shows the size of the matrices for identification using ERA and LIM in the NPCC system in simple identifications of 24 inputs ( $\mathrm{Vr}$ or $\mathrm{Pm}$ ) and multiple identification grouping measurements obtaining 48 inputs $(\mathrm{Vr}$ and $\mathrm{Pm})$, for all identification methods the same energy criteria are used, $E_{\text {era }}=E_{\text {lim }}=92 \%$ and $\alpha_{i}=0.01$.

Fig. 7 shows the eigenvalues of the identified system with different methods. Figs. 7(a) and 7(b) show the results for the case of individual measurements, 
Table 4. RMSE of NPCC system identification by increasing the amplitude of the probing signal.

\begin{tabular}{c|c|c|c|c|c}
\hline Method & $\alpha=0.05$ & $\alpha=0.14$ & $\alpha=0.23$ & $\alpha=0.32$ & $\alpha=0.41$ \\
\hline ERA & $0.63 \times 10^{-3}$ & $0.72 \times 10^{-3}$ & $4.02 \times 10^{-3}$ & $18.25 \times 10^{-3}$ & $23.32 \times 10^{-3}$ \\
LIM & $0.58 \times 10^{-3}$ & $0.67 \times 10^{-3}$ & $1.81 \times 10^{-3}$ & $2.39 \times 10^{-3}$ & $2.87 \times 10^{-3}$
\end{tabular}

Table 5. ERA and LIM matrices in NPCC system.

\begin{tabular}{c|c|c|c|c|c|c}
\hline Mod. & $\mathbb{L}_{r}, \sigma \mathbb{L}_{r}$ & $\mathbb{F}_{r}$ & $\mathbb{W}_{r}$ & Order & $\tilde{\mathbf{H}}(1), \tilde{\mathbf{H}}(2)$ & Order \\
\hline $\mathrm{Vr}$ & $480 \times 240$ & $480 \times 24$ & $48 \times 240$ & 49 & $9600 \times 4800$ & 56 \\
\hline $\mathrm{Pm}$ & $480 \times 240$ & $480 \times 24$ & $48 \times 240$ & 40 & $9600 \times 4800$ & 47 \\
\hline $\mathrm{Vr}+\mathrm{Pm}$ & $480 \times 480$ & $480 \times 48$ & $48 \times 480$ & 76 & $9600 \times 9600$ & 61 \\
\hline
\end{tabular}

$\mathrm{Vr}$ and Pm, respectively. Fig. 7(c) shows the same result but for the case of multiple measurements $\mathrm{Vr}$ and Pm (together). Note that for all cases the accuracy of the local and inter-area mode identified is high in the selected frequency band. However, when combining the measurements there is a higher density of modes identified close to in the actual modes (SSA).

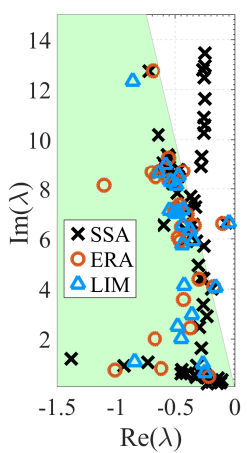

(a) Simple $\mathrm{Vr}$

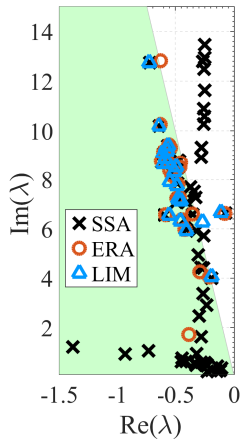

(b) Simple Pm

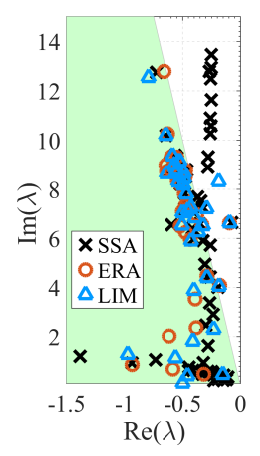

(c) Multiple Vr, Pm
Figure 7. NPCC eigenvalues grouping measurements.

In Fig. 8 shows the Bode diagram of identified linear systems, using single and multiple data, in Fig. 8(a) shows $\omega_{1} / V r_{1}$ and in Fig. 8(b) shows $\omega_{1} / P m_{1}$.

Table 6 presents the RMSE for the identified systems, $\omega_{1} / V r_{1}$. Using LIM the error obtained is $0.57 \times 10^{-3}$, which $53 \%$ less than the one obtained with ERA. Similarly, $\omega_{1} / P m_{1}$ presents an error $0.54 \times 10^{-3}$ which is $58 \%$ less than the value obtained with ERA.

Table 6. Identification accuracy in NPCC system.

\begin{tabular}{c|c|c|c}
\hline Transfer function & Method & Simple & Multiple \\
\hline \multirow{2}{*}{$\omega_{1}(s) / V r_{1}(s)$} & ERA & $1.20 \times 10^{-3}$ & $1.20 \times 10^{-3}$ \\
\cline { 2 - 4 } & LIM & $0.95 \times 10^{-3}$ & $0.57 \times 10^{-3}$ \\
\hline \multirow{2}{*}{$\omega_{1}(s) / P m_{1}(s)$} & ERA & $1.08 \times 10^{-3}$ & $1.13 \times 10^{-3}$ \\
\cline { 2 - 4 } & LIM & $1.19 \times 10^{-3}$ & $0.54 \times 10^{-3}$ \\
\hline
\end{tabular}
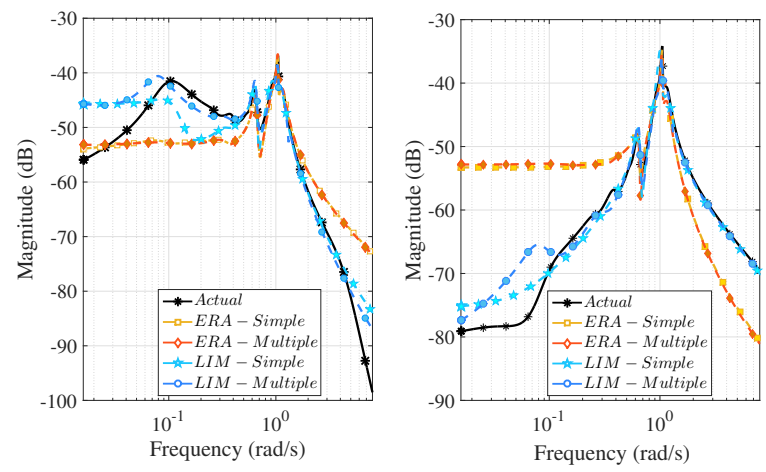

(a) $\omega_{1} / V r_{1}$ Simple and Multiple (b) $\omega_{1} / P m_{1}$ Simple and Multiple

Figure 8. NPCC precision of ERA and LIM methods by grouping measurements.

\section{Conclusions and Future Work}

This paper compares the ERA and LIM methods for estimating linear representation of power systems in different scenarios. The results of the paper show that the LIM method has a greater precision than the ERA method for identifying the linear model of a power network. This result is held even in the presence of noise produced by the nonlinear characteristics of the system. This is mainly due to the fact that the ERA method uses a frequency sweep signal and has a harder time obtaining frequency components in the presence of noise. This is in contrast of the LIM method which uses a more robust time-invariant signal to extract the frequency response of the system.

This paper applies the ERA and LIM methods to the KRK and NPCC test systems for system identification under different conditions. The results presented here verify that the LIM method has the advantage of considerably improving its accuracy by grouping the measurements (combining modulations of different inputs). This allows reducing the number of frequencies used in the identification as well as the time of the transitory simulations.

Future work includes the use of LIM method with signals that excite a greater number of modes, such as cosine or linear chirp, and therefore reduce the modulation time of the input system which can help in the the implementation of this method in real time. Likewise, it is possible to change the method of the 
Fast Fourier Transform (FFT) for other more advanced methods such as the Taylor-Fourier Transform (TFT) or B-Splines to increase the accuracy in calculating the frequency components.

\section{Acknowledgment}

Sandia National Laboratories is a multi-mission laboratory managed and operated by National Technology and Engineering Solutions of Sandia, LLC., a wholly owned subsidiary of Honeywell International, Inc., for the U.S. Department of Energy's National Nuclear Security Administration under contract DE-NA0003525.

\section{References}

[1] F. TP462, "Identification of electromechanical modes in power systems," IEEE Task Force Report, 2012.

[2] J. J. Sanchez-Gasca and J. H. Chow, "Performance comparison of three identification methods for the analysis of electromechanical oscillations," IEEE Transactions on Power Systems, vol. 14, no. 3, pp. 995-1002, 1999.

[3] R. H. Byrne, R. J. Concepcion, J. Neely, F. Wilches-Bernal, R. T. Elliott, O. Lavrova, and J. E. Quiroz, "Small signal stability of the western north american power grid with high penetrations of renewable generation," in 2016 IEEE 43rd photovoltaic specialists conference (PVSC), pp. 1784-1789, IEEE, 2016.

[4] J.-N. Juang and R. S. Pappa, "An eigensystem realization algorithm for modal parameter identification and model reduction," Journal of guidance, control, and dynamics, vol. 8, no. 5, pp. 620-627, 1985.

[5] I. Kamwa, R. Grondin, J. Dickinson, and S. Portia, "A minimal realization approach to reduced-order modelling and modal analysis for power system response signals," IEEE Transactions on Power Systems, vol. 8, pp. 1020-1029, aug 1993.

[6] L. D. Peterson, "Efficient computation of the eigensystem realization algorithm," Journal of Guidance, Control, and Dynamics, vol. 18, no. 3, pp. 395-403, 1995.

[7] G. Gurrala, "Loewner matrix approach for modelling fdnes of power systems," Electric Power Systems Research, vol. 125, pp. 116-123, 2015.

[8] A. Mayo and A. Antoulas, "A framework for the solution of the generalized realization problem,"
Linear algebra and its applications, vol. 425, no. 2-3, pp. 634-662, 2007.

[9] M. Kabir and R. Khazaka, "Macromodeling of distributed networks from frequency-domain data using the loewner matrix approach," IEEE Transactions on Microwave Theory and Techniques, vol. 60, no. 12, pp. 3927-3938, 2012.

[10] S. Lefteriu and A. C. Antoulas, "A new approach to modeling multiport systems from frequency-domain data," IEEE Transactions on Computer-Aided Design of Integrated Circuits and Systems, vol. 29, no. 1, pp. 14-27, 2010.

[11] C. M. Rergis, I. Kamwa, R. Khazaka, and A. R. Messina, "A Loewner Interpolation Method for Power System Identification and Order Reduction," IEEE Transactions on Power Systems, vol. 34, pp. 1834-1844, may 2019.

[12] J. S. Bay, Fundamentals of linear state space systems. McGraw-Hill, 1999.

[13] F. Wilches-Bernal, R. H. Byrne, and J. Lian, "Damping of inter-area oscillations via modulation of aggregated loads," IEEE Transactions on Power Systems, vol. 35, no. 3, pp. 2024-2036, 2020.

[14] M. Kabir, R. Khazaka, R. Achar, and M. Nakhla, "Loewner-matrix based efficient algorithm for frequency sweep of high-speed modules," in 2012 IEEE 21st Conference on Electrical Performance of Electronic Packaging and Systems, pp. 185-188, IEEE, 2012.

[15] Y. Wang, C.-U. Lei, G. K. Pang, and N. Wong, "Mfti: Matrix-format tangential interpolation for modeling multi-port systems," in Design Automation Conference, pp. 683-686, IEEE, 2010.

[16] M. Klein, G. J. Rogers, and P. Kundur, "A fundamental study of inter-area oscillations in power systems," IEEE Transactions on Power Systems, vol. 6, no. 3, pp. 914-921, 1991.

[17] D. Osipov and K. Sun, "Adaptive Nonlinear Model Reduction for Fast Power System Simulation," Nov 2017.

[18] J. H. Chow and K. W. Cheung, "A toolbox for power system dynamics and control engineering education and research," IEEE Transactions on Power Systems, vol. 7, no. 4, pp. 1559-1564, 1992.

[19] J. H. Chow, R. Galarza, P. Accari, and W. W. Price, "Inertial and slow coherency aggregation algorithms for power system dynamic model reduction," IEEE Transactions on Power Systems, vol. 10, no. 2, pp. 680-685, 1995. 